\title{
Flow cytometric evaluation of the effects of 3-bromopyruvate (3BP) and dichloracetate (DCA) on THP-1 cells: a multiparameter analysis
}

\author{
Harrie A. Verhoeven • Leo J. L. D. van Griensven
}

Received: 27 December 2011 / Accepted: 12 January 2012 / Published online: 9 February 2012

(C) The Author(s) 2012. This article is published with open access at Springerlink.com

\begin{abstract}
Two human leukemia cells K562 and THP-1, the breast cancer lines MCF-7 and ZR-75-1, and the melanoma line MDA-MB-435S were compared by flowcytometry for their behaviour at increasing levels of 3BP. K562 and THP-1 responded to 3BP by membrane depolarization and increased ROS; MCF-7 and ZR-75-1 showed decreased polarization and low ROS increase; MDA-MB-435S had limited depolarization and no ROS increase. THP-1 cells exposed to a range of 3BP concentrations in combination with DCA showed increase of polarization, slight ROS increase, and weakened nuclear integrity. 3BP and DCA show no synergism, but have complementary destructive effects on THP-1 cells. The data led to the conclusion that the THP-1 cells do not carry a functional membrane monocarboxylate transporter (MCT) or that 3BP circumvents MCT binding and can enter these cells independently.
\end{abstract}

Keywords 3-bromopyruvate - DCA - THP-1 . Flowcytometry $\cdot$ Cancer $\cdot$ ROS $\cdot$ Membrane polarization . MCT-1

\section{Introduction}

The past 30 years have seen a dramatic increase in the number of drugs for the treatment of cancer. This development has been spurred by the rapid developments in the area

H. A. Verhoeven $(\varangle) \cdot$ L. J. L. D. van Griensven

Bioscience, Plant Research International,

Wageningen University and Research Centre,

POB 16, 6700AA Wageningen, the Netherlands

e-mail: harrie.verhoeven@wur.nl of molecular biology, biochemistry and chemical synthesis, resulting in ever more complex drugs that can be tested for their biological efficacy. The growing insight in (sub) cellular communication pathways, involving complex regulation cascades has provided an overwhelming number of drugs intended to disrupt the cellular processes that allow tumors to thrive and destroy their host. Unfortunately, cancer cells have the ability to evade the very specific action of many of these new drugs, either by circumventing their accumulation in their cells by a series of transporters, aimed at eliminating these drugs from the cells interior, or by escaping their action by mutations on the active sites of the enzymes which are the target of these drugs. In combination with severe deleterious effects of most of these drugs on the essential processes for the patient, this results in a heavy burden to the patients who undergo these treatments. In order to extend the range of useful drugs, new insights are needed.

85 years ago, a common feature of the cells comprising solid tumors was discovered by Warburg: aerobic glycolysis (Warburg et al. 1926). In contrast to normal glycolysis, this process is able to run under aerobic conditions, and provides the tumor cells with a set of unique properties. The first step in this process is catalyzed by the enzyme hexokinase, and most solid tumors have isoform 2 overexpressed. This enzyme is the basis for the application of ${ }^{18}$ F-deoxyglucose as a tracer for the localization of malignant cells in a PET-scan. This sugar analog is taken up by all cells, but only hexokinase 2 (HK2) is able to phosphorylate it into a form which cannot be metabolized further due to the deoxy character of this sugar, resulting in accumulation in malignant cells, thereby enabling the localisation of these cells by looking for the annihilation radiation of the emitted positron. The relatively low energy of the positron improves localization resolution. 
Hexokinase 2 is bound to the voltage dependent anion channel (VDAC) complex of the mitochondria, thus causing hyperpolarization and inhibiting apoptosis and cell death (Chen et al. 2009).

3-Bromopyruvate (3BP) has been shown to eradicate liver tumors in an animal test system (Ko et al. 2004; Geschwind et al. 2002) and it has been shown to inhibit HK2 by binding covalently in the active site of the enzyme by alkylation of a cysteine residue (Porporato et al. 2011). In addition, it inhibits a number of other enzymes from the glycolytic pathway, such as glyceraldehyde-3-phosphate dehydrogenase, and succinate driven ATP synthesis (Dell'Antone 2009).

Dichloroacetate (DCA) is a pyruvate dehydrogenase kinase inhibitor which leads to increased pyruvate dehydrogenase activity (Papandreou et al. 2011). This causes in cells increased conversion of pyruvate to acetyl-CoA rather than lactic acid, and stimulates mitochondrial respiration by increasing the supply of acetyl-CoA. Although DCA is not a specific inhibitor of tumor cell glycolysis (Stockwin et al. 2010), it may be used to target cells that show defects in the mitochondrial electron transport chain. DCA could have a synergistic activity with anti-cancer drugs that impair mitochondrial activity. It has been used to inhibit growth of tumor cells in vitro (Sun et al. 2010) as well as in vivo (Michelakis et al. 2010). To study the effects of 3BP and DCA individually as well as combined on tumor cell growth in vitro we used 2 different leukemia derived cell lines, i.e. the monocytic leukemia line THP-1 (Tsuchiya et al. 1980) and the erythroleukemia line K562 (Lozzio and Lozzio 1975), and 3 cell lines derived of solid tumors, the breast tumor lines MCF-7 (Soule et al. 1973), ZR-75-1 (Engel et al. 1978) and the melanoma line MDA-MB-435S (Ellison et al. 2002).

In this paper we present data on the comparison of the effects of $3 \mathrm{BP}$ on various tumor derived human cell lines, along with data from combined treatments of the THP-1 monocyte derived cell line. The mechanisms involved in the activation of ROS species and membrane depolarization are discussed, with emphasis on the presumed role of membrane monocarboxylate transporters (MMCT) in internalizing 3BP.

\section{Materials and methods}

Cells and chemicals

Cells were obtained from the following sources. THP1 cells were obtained from Cell Lines Service, Eppelheim, Germany; K562 were kindly provided by Prof. H.F.J. Savelkoul, Dept. Cell biology and Immunology, Wageningen UR; the other cell lines were provided by
Dr. Young Ko, Johns Hopkins University, School of Medicine, Baltimore, USA. All cells were grown in RPMI 1640 culture medium (Sigma, cat R8758) supplemented with 10\% heat-treated newborn calf serum (Gibco, cat. 161010-159) and penicillin/streptomycin (Sigma-Aldrich) at $37^{\circ} \mathrm{C}$ in a humidified incubator at $5 \% \mathrm{CO} 2$.

3BP, dihydroethidium, propidium iodide and other chemicals were obtained from Sigma-Aldrich, except JC1, which was obtained from Enzo Life Sciences (cat. Enz-52304),

\section{Cell culture}

THP-1 and K562 cells were plated in 24 well plates (Costar) with cell density of $2 \times 10^{5}$ cells resp. $4 \times 10^{5}$ cells per well. The adherent cell lines were plated 4 days before the experiment and used when $80 \%$ confluent. After $24 \mathrm{~h}$ cultures of THP-1 and K562 were used directly for staining and flowcytometry purposes. The adherent cultures were first trypsinized and cells were washed in complete medium and collected by centrifugation. For 3BP and DCA treatment respectively a solution of $10 \mathrm{mM} 3 \mathrm{BP}$ was freshly made in PBS and sterilized by ultrafiltration, while $1 \mathrm{M}$ DCA was prepared in water, brought to $\mathrm{pH} 7$ with $\mathrm{NaOH}$, sterilized by ultrafiltration and if necessary stored frozen.

\section{JC-1 staining}

JC-1 staining for determining mitochondrial membrane polarization was carried out by adding $4 \mu \mathrm{g} / \mathrm{ml} \mathrm{JC}-1$ to the medium after which the cells were directly used for flowcytometry.

\section{Dihydroethidium (DHE) staining}

DHE staining for determining the amount of reactive oxygen species was carried out by adding $100 \mathrm{ng} / \mathrm{ml}$ DHE to the medium after which the cells were directly used for flowcytometry.

Propidium iodide (PI) staining

Cells were collected by centrifugation and suspended in PBS. RNA was degraded by adding $20 \mu \mathrm{g} / \mathrm{ml}$ RNase A (DNase-free) and incubating for $1 \mathrm{~h}$ at room temperature. Cells were then stained by adding 1 volume of $0.1 \%$ Triton X100 in PBS and $10 \mu \mathrm{g}$ PI per ml. Cells were then immediately used for flowcytometry.

\section{Effect of N-Acetylcysteine}

Cells were incubated at $37{ }^{\circ} \mathrm{C}$ with $0,10,20$, and $50 \mu \mathrm{M} \mathrm{N}$ Acetylcysteine (NAC) in a 24 well plate in a matrix setting 
with $0,10,20$, and $50 \mu \mathrm{M} 3 \mathrm{BP}$ and harvested after $24 \mathrm{~h}$. NAC treatment was started $1 \mathrm{~h}$. before the addition of $3 \mathrm{BP}$ in order to saturate cells with NAC.

\section{Flow cytometry}

A DAKO Cyan instrument was used for flowcytometric analysis, equipped with a 488 solid state blue green laser, operated at $10 \mathrm{~mW}$ output power. The standard filter/dichroic mirror set was used, and 4 fluorescence, forward scatter and sideward scatter signals were recorded, in both logarithmic and linear mode.

Data were collected from the samples during a run of $30 \mathrm{~s}$ per sample. In this way, about 1,500 to 6,000 cells were analyzed during every run, allowing a comparison of cell growth or toxic effects of the treatments. Experiments were performed in duplo, with one replicate stained with JC1 and the other with DHE. In one experiment also DNA amount was determined by PI staining. Gating was applied in the forward/sideward scatter diagram to select for intact cells.

\section{Data analysis}

Data were recorded in fcs 3 file format and analysed using the free version of Cyflogic, version 1.2.1 (www.cyflogic.com). Selected data were exported to Excel for statistical analysis, using the StatistiXL version 1.8 (www.statistiXl.com) plug in. The casewise PCA scores were plotted as round dots in graphs with the relevant PC's on the horizontal and vertical axis. Component Score Coefficients (Eigenvectors) were indicated in these graphs with small squares as endpoint.

\section{Results}

Cell line comparison

In total, 5 different human cell lines were tested for their behaviour at increasing levels of 3BP. For this purpose, the following parameters were extracted from the flowcytometry data files: Median intensity of fluorescence intensity of $\mathrm{JC} 1$ resp. DHE staining, together with the $\mathrm{CV}$ of the distribution, median of the forward and sideward scatter signal, and median and gated signal of the pulse time, in order to discriminate between single cells and clusters.

The PCA plot (all parameters normalized to 1SD) of these data (from one experiment) is represented in Fig. 1. From this diagram can be concluded that all cell lines show clear differences in the parameters extracted from the data. The horizontal axis ( $\mathrm{PC} 1,45.8 \%$ of variance) differentiates between the strongly adherent cells on the left (MCF-7 and ZR-75-1), which show a large amount of clustering (35 to $50 \%$ doublets), indicated by the higher pulse time (vector PTmean jc1), when compared to the single cells of THP-1, K562 and MDA-MB-435S cells (5 to 10\% doublets). The arrows within the ellipses indicate the direction of the effects of increasing 3BP concentrations, with the arrowhead representing $500 \mu \mathrm{M}$ 3BP. The inversion point, marked with a diamond, indicates the highest concentration of 3BP that does not result in massive cell death $(50 \mu \mathrm{M}$ for THP-1, and ZR-75-1, $100 \mu \mathrm{M}$ for MCF-7, and no limit for K562).

These diagrams also reveal strong differences in the cellular response to increasing 3BP concentrations. The K562 and THP-1 lines respond both by membrane depolarization and
Fig. 1 PCA plot of the comparison of the response to increasing concentrations of $3 \mathrm{BP}$ of 5 different cell lines. Concentrations are indicated at each round dot, representing one experiment. The lines ending with squares are the eigenvectors of the individual measured parameters, quantified from the flowcytometry data. For clarity, only selected parameters were labelled. The arrows indicate the increasing 3BP concentrations $(0,10,20,50$, 100 and $500 \mu \mathrm{M}$ ), with the arrowhead at $500 \mu \mathrm{M}$. The inversion point (diamond) indicates the highest tolerated 3BP concentration, marked in all cases, except K562

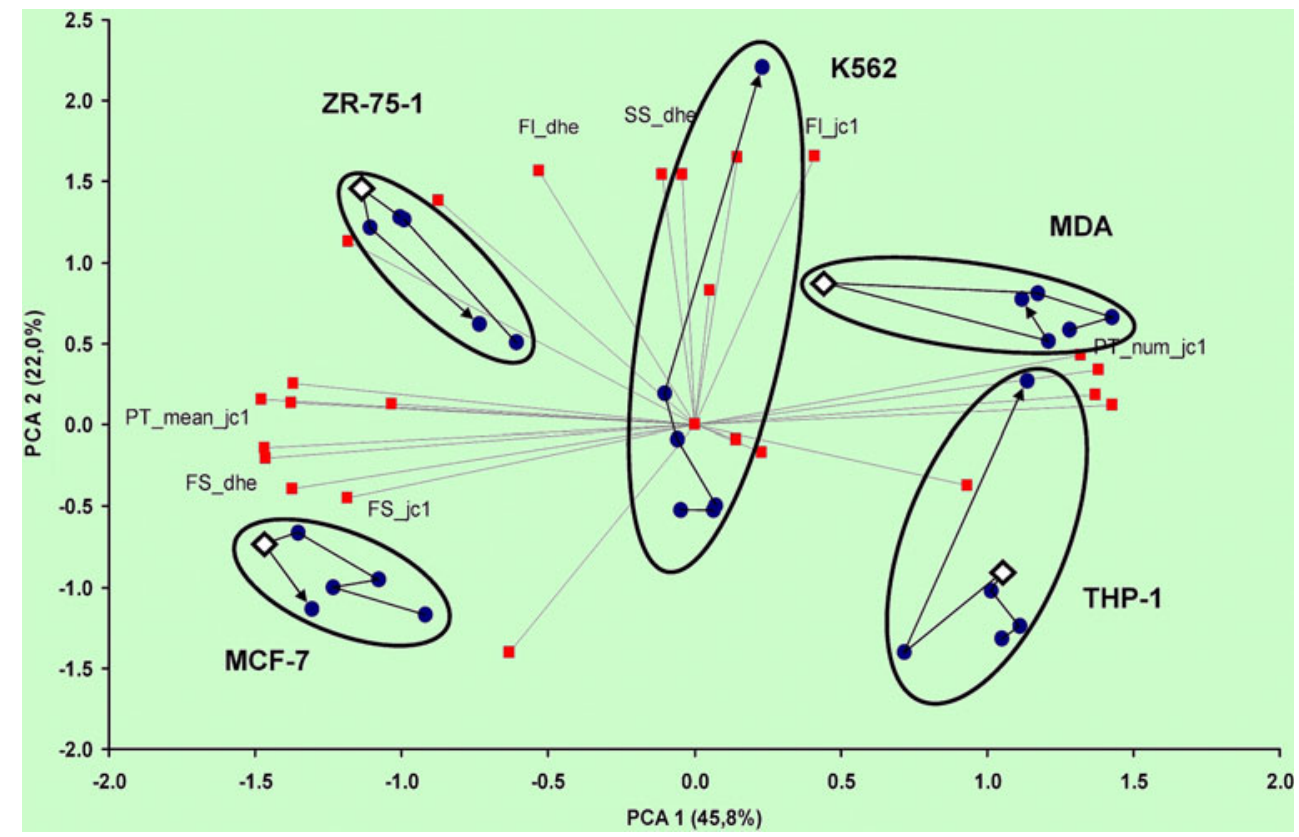


increased ROS production, as shown by their orientation parallel to the vectors representing the Fl_jcl and Fl_dhe signals for JC1 and DHE respectively. The ZR-75-1 and MCF-7 do respond with a decrease of their membrane potential, but ROS production is much less pronounced than with THP-1 and K562. The same holds true for MDA-MB-435S, which did not respond with any ROS production increase and very limited membrane depolarization. The MDA-MB-435S line shows its strongest response in the forward scatter signal (vector FS_dhe), indicating that cells respond by increasing size with increasing $3 \mathrm{BP}$ concentrations. This is also true, but to a lesser degree with MCF-7 and ZR-75-1.

These aspects are illustrated in Fig. 2, which shows a selection of the most discriminant features detected in the flowcytometry data. Each cell line has a distinct pattern in the combined forward/sideward scatter plot (left column). The gating of the cells for detection of the fluorescence profiles is accommodated accordingly to the different scatter properties of each cell line. The pulse time histograms reveal the excessive clustering (50\%) of cells in the MCF-7 and less in the ZR$75-1$ cell lines (35\%) (indicated by the solid arrows). The JC1 and DHE fluorescence distributions presented are from the 0BP (solid line) and that concentration of 3BP which gave the first change of membrane potential, the concentration being marked to the left of the diagrams. The MCF-7 cells did react with membrane depolarization at $100 \mu \mathrm{M} 3 \mathrm{BP}$, but did not show any increase of ROS at this concentration. At $200 \mu \mathrm{M}$, the MDA-MB-435S exhibited the first signs of membrane depolarization and a shift to higher ROS production. The ZR-75-1 cells did react to $50 \mu \mathrm{M} 3 \mathrm{BP}$ but showed a drastic effect at $100 \mu \mathrm{M}$ as shown in Fig. 2. THP-1 has a consistent effect in all our experiments which starts at $20 \mu \mathrm{M}$ and results in massive cell damage at $50 \mu \mathrm{M}$. No effect could be noted on K562 cells until the concentration reached $500 \mu \mathrm{M}$. At this concentration, the resistance of this cell line to 3BP collapses dramatically, with rapid membrane depolarisation and ROS increase.

Effects of combined 3BP and DCA treatments on THP- 1 cells

Cells were incubated in 24 well plates for $24 \mathrm{~h}$ at different concentrations of $3 \mathrm{BP}$ and DCA and a combination of both i.e. $0,10,20,50 \mu \mathrm{M} 3 \mathrm{BP}$ and $0,2,5,10,20,50 \mathrm{mM}$ DCA. Although the highest concentration of both compounds caused obvious cell death of THP-1 cells as determined by strongly increased trypan blue uptake in the nuclei (data not shown), no prevalence of apoptosis was observed in PI stained cell preparations (data not shown).

The effects of the various combinations of 3BP and DCA are summarized in Fig. 3. This PCA plot (with data from one experiment) shows the control in the upper right hand corner (marked 0BP) and increasing concentrations of DCA $(2,5,10,20$ and $50 \mathrm{mM})$ are connected with solid lines, with $50 \mathrm{mM}$ at the arrowhead. The dashed lines are connecting points with identical concentrations of the increasing 3BP concentrations at the various DCA concentrations, with the arrowhead indicating $50 \mu \mathrm{M} 3 \mathrm{BP}$.

The effect of DCA is mainly an increase of membrane depolarization, while only slightly increasing ROS activity. In addition, increasing DCA concentrations have a strong effect on both the median and the CV of the PI signal (vectors Fl_pi and CV_pi respectively), suggesting an effect on nuclear integrity. To a lesser degree an effect is visible on the sideward scatter signals (vectors SS_dhe and SS_jc1), which would support this observation. Increasing $3 \mathrm{BP}$ concentrations finally leads to a strong reduction of membrane potential and strong induction of ROS activity, indicated by the cluster with all $50 \mu \mathrm{M} 3 \mathrm{BP}$ treatments in the left part of the diagram (dotted ellipse).

The data from all combination experiments with DCA, and two combinations with the addition of lactate, are summarized in Fig. 4. Since variation occurs in between experiments in the intensity of the $\mathrm{JC} 1$ and DHE staining, caused by fluctuations in cell viability between batches of cells, a different approach was taken for the processing of these results. The median of the JC1 and DHE fluorescence distributions of sample of 0 $\mathrm{BP}$ without additions was chosen as reference, and all other median fluorescence values were expressed as the ratio of the sample with the reference. All relative values were then averaged over the different experiments. Due to the number of samples that could be processed in a limited time, not all experiments did encompass all combinations tested. The points with $0 \mathrm{BP}$ are indicated with open symbols, and each line is labelled with the concentration of DCA or lactate (LAC) used.

This figure shows the strong induction of ROS activity (about 10 times), along with membrane depolarization due to 3BP alone (2 times), or in combination with DCA and lactate. The effect of DCA is most clearly on the membrane depolarization, with a slight reduction (halving) in ROS stimulation by $3 \mathrm{BP}$, which is most apparent in the $5 \mathrm{mM}$ DCA samples. The graphs from the 10 and $20 \mathrm{mM}$ DCA treatments show similar effects on membrane depolarisation and ROS induction, indicating a saturation effect when compared to 2 and $5 \mathrm{mM}$ DCA.

The $50 \mathrm{mM}$ DCA samples show a reduced membrane depolarisation which is caused by the damaged membranes of the cells and a strong reduction in cell numbers (data not shown). The slope of the lines connecting the $03 \mathrm{BP}$ treatments and the $50 \mu \mathrm{M} 3 \mathrm{BP}$ treatments for 0 to $5 \mathrm{mM}$ DCA are virtually parallel, indicating a lack of synergy of these two treatments, but rather pointing towards complementary effects. Higher concentrations of 


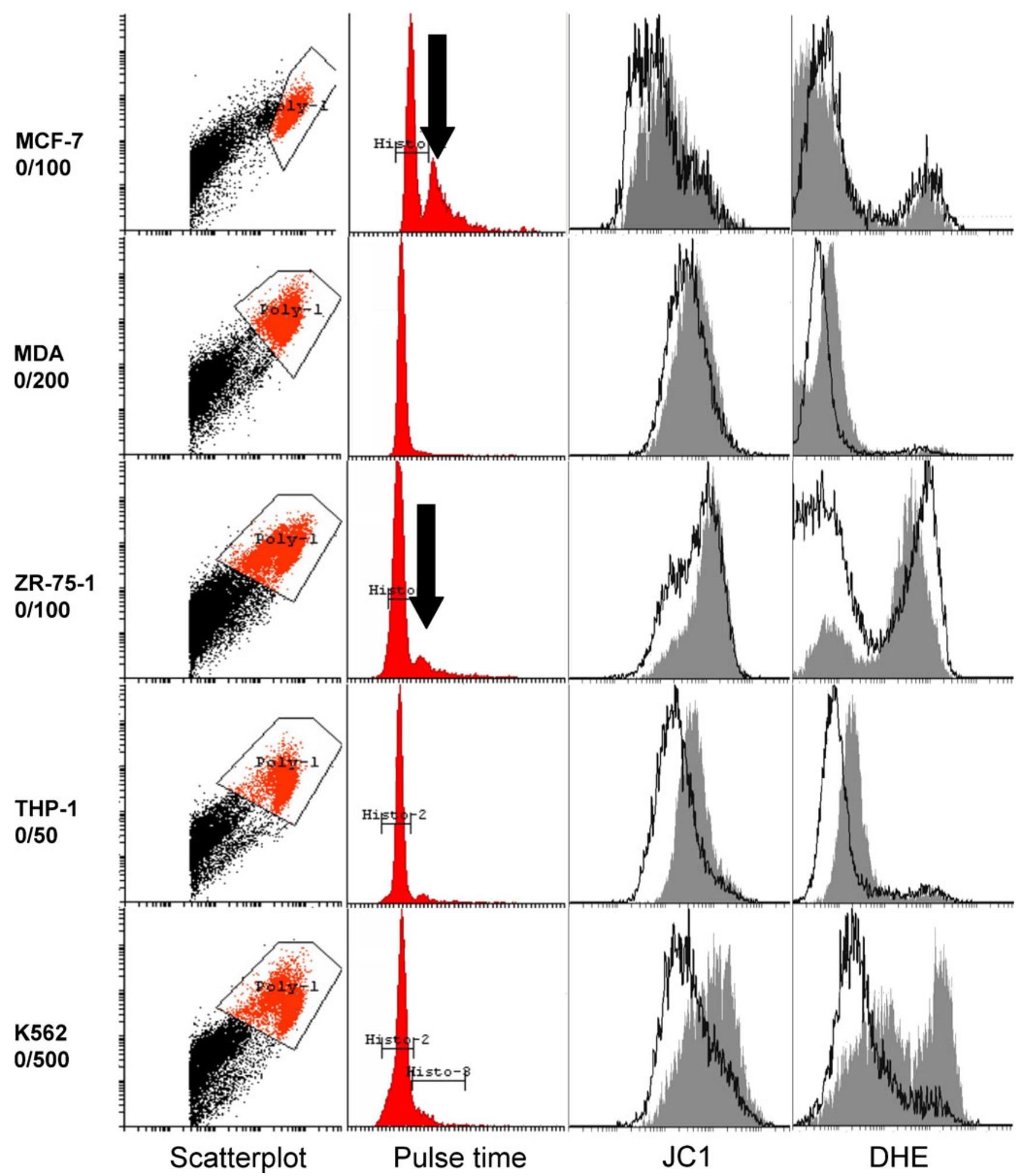

Fig. 2 Typical details from the flowcytometric data of each cell line. Details were selected according to the PCA plot to show the most discriminant features at the concentration of 3BP which inflicted the most effects on each cell line. The scatterplots show the combined forward (horizontal axis) and sideward (vertical axis) scatter, along with the area used for gating. The pulse time histogram(horizontal axis) of the gated cells is shown to indicate the presence of clusters in the
MCF-7 and ZR-75-1 cell lines (solid arrows), together with the quantification windows histo-2 and histo-3. For both JC1 and DHE fluorescence intensity histograms (fluorescence intensity on the horizontal axis), the zero BP (solid line), as the 3BP concentration (indicated at the left following the / as $\mu \mathrm{M}$ ) at which the most drastic changes were identified are given as grey shaded area 
Fig. 3 PCA plot of selected parameters from the cells, analysed using JC1, DHE and $\mathrm{PI}$ in combination with RNase treatment. The eigenvectors of the individual parameters are represented by the lines and marked by the name of the parameter. For clarity only selected parameters were labelled. Control cells are located in the upper right corner of the plot (at the dot labelled $\mathrm{BP} 0$, whereas the $50 \mu \mathrm{M} 3 \mathrm{BP}$ treated samples are in the left of the diagram within the dotted ellipse. Corresponding treatments are connected via arrows (3BP ranges) or dotted arrows (DCA ranges), with the arrowhead marking the highest $3 \mathrm{BP}(50 \mu \mathrm{M})$ and DCA $(50 \mathrm{mM})$

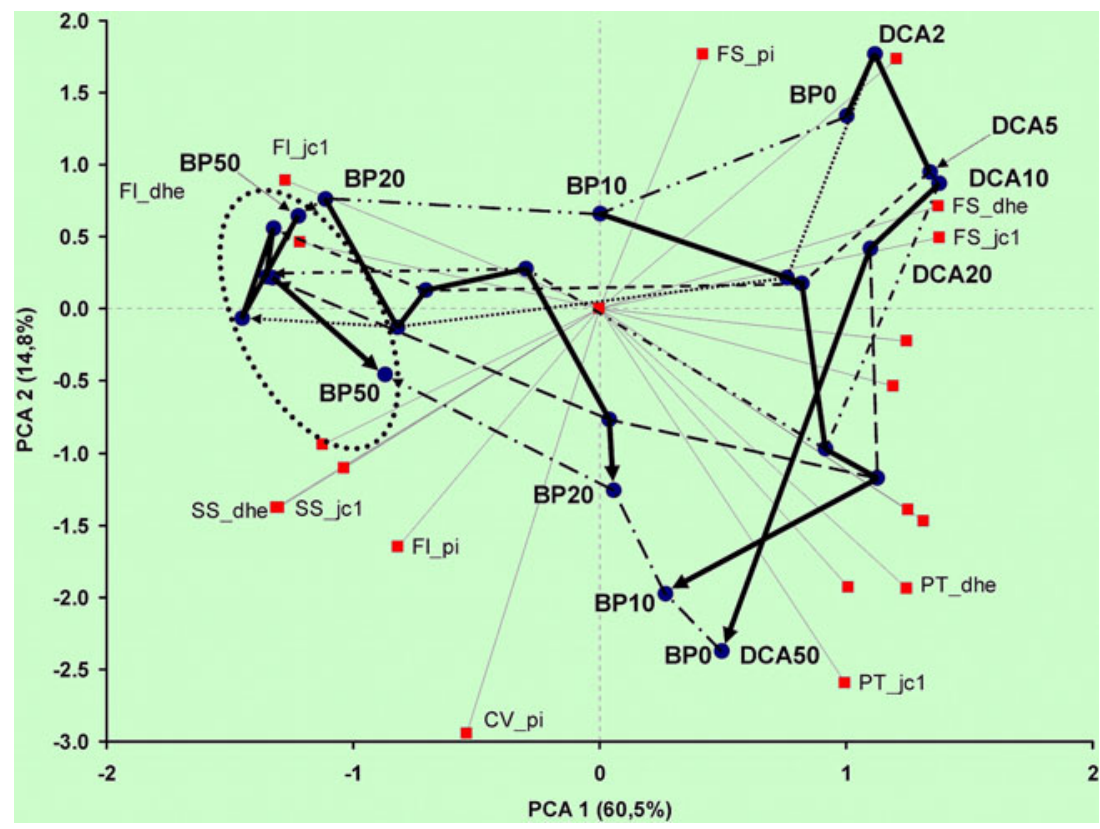

DCA show an increase in ROS activity but without increase of membrane polarization.

The combinations with lactate show a reduced (about $60 \%$ reduction) increase of ROS activity by increasing $3 \mathrm{BP}$ concentration which is comparable to the effect of the $5 \mathrm{mM}$ DCA combination. The effect of increasing 3BP

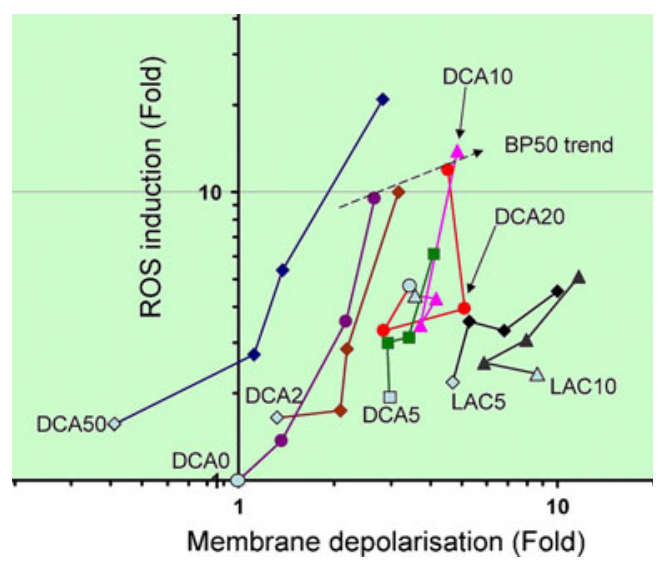

Fig. 4 Double logarithmic representation of the average ratios of median values of JC1 and DHE fluorescence of combinations of 3BP $(0,10,20$ and $50 \mu \mathrm{M})$ with DCA $(0,2,5,10,20$ and $50 \mathrm{mM})$ and lactate (LAC) (2 and $5 \mathrm{mM}$ ) (Pooled from 7 independent experiments). The 0 BP (Marked with an open symbol) samples are indicated. The arrows represent the trend of the $50 \mu \mathrm{M} 3 \mathrm{BP}$ treatment. The zero BP sample was taken as reference, therefore its location at coordinate (1, 1). The units along the axis indicate fold increase or decrease due to increasing 3BP concentrations in combinations with DCA and lactate. Number of experiments: DCA $0 \mathrm{mM}$ : 7; DCA $2 \mathrm{mM}$ : 3; DCA $5 \mathrm{mM}$ : 6; DCA10mM: 6; DCA $20 \mathrm{mM}$ : 5; DCA $50 \mathrm{mM}$ : 2; lactate $2 \mathrm{mM}: 2$ and lactate $5 \mathrm{mM}: 2$ concentrations in combination with lactate leads to an even stronger membrane depolarization than when compared to DCA or $3 \mathrm{BP}$ alone.

Effects of combined treatments with 3BP and NAC

The results of this experiment are shown in the PCA plot in Fig. 5. This figure reveals a complicated interaction between $3 \mathrm{BP}$ and NAC. At lower concentrations of 3BP (up to $20 \mu \mathrm{M}$ ), NAC is able to compensate the membrane depolarization induced by $3 \mathrm{BP}$ at 10 and $20 \mu \mathrm{M}$ NAC. Higher concentrations of NAC (50 and $100 \mu \mathrm{M})$ have their own effect on cellular parameters, the most obvious one being Forward scatter (vector FS_dhe), indicating an increase of cell size. Only $50 \mu \mathrm{M} 3 \mathrm{BP}$ (BP50) induces a strong increase of ROS (3X, vector Fl_dhe) and increasing concentrations of NAC (NAC10, NAC20 and NAC50) strongly affect the ROS induction in a dose dependent way, resulting in a strong reduction at $50 \mu \mathrm{M}$.

\section{Discussion}

It remains a mystery why the glycolytic nature of the energy supply of solid tumors has escaped research efforts for so long. In this paper we present data about the effect of 3BP, assumed to be an inhibitor of HK2, the first dedicated enzymatic step in aerobic glycolysis of tumor cells (Ko et al. 2001; Pedersen 2007) on different human cell lines, derived from solid tumors, (MCF-7, ZR-75-1, MDA-MB435S) and the suspension culture lines, THP-1 and K562, 


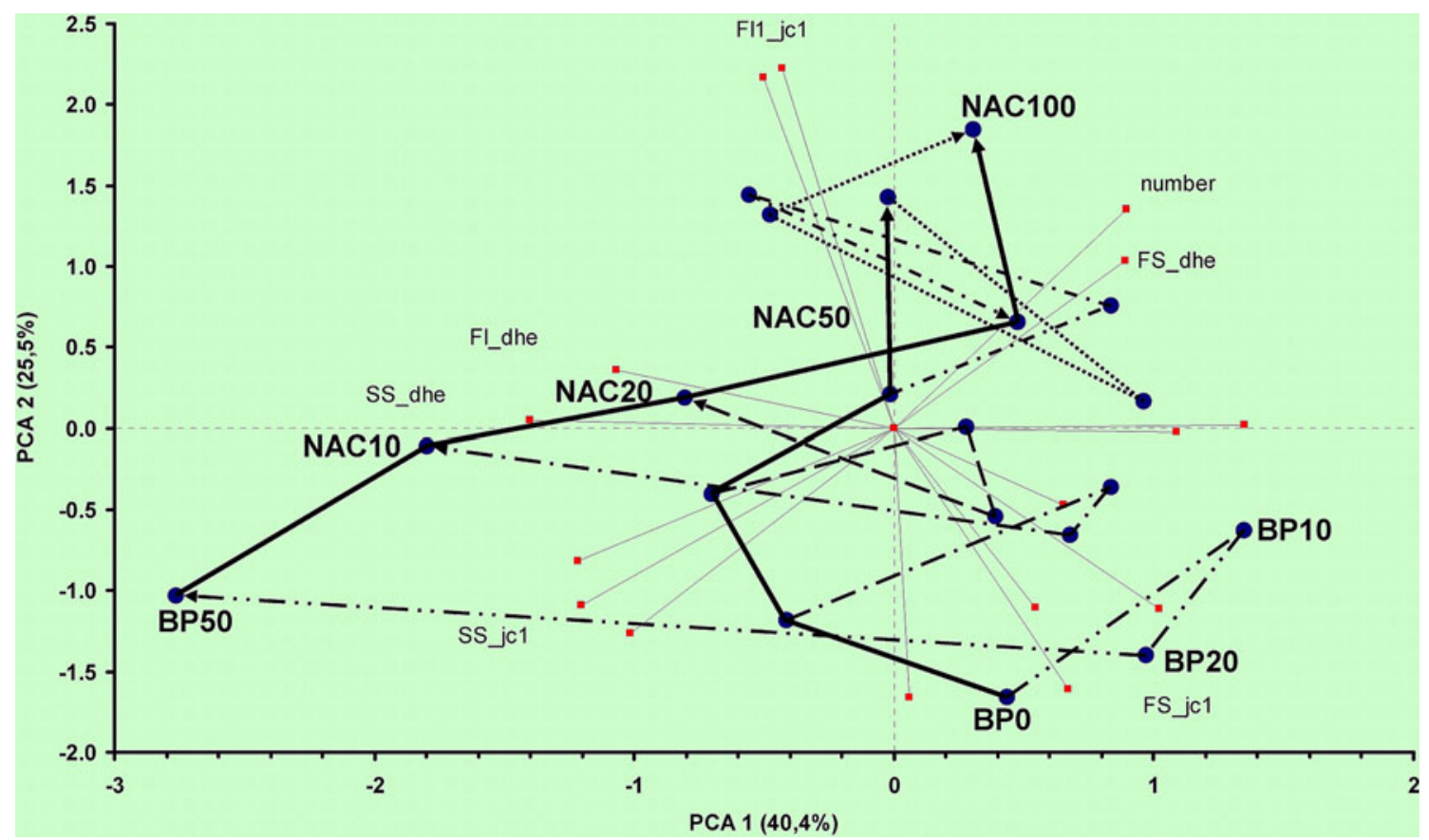

Fig. 5 PCA plot of a combination experiment of varying $3 \mathrm{BP}(0,10$, 20 and $50 \mu \mathrm{M})$ concentrations with varying NAC concentrations $(0$, $10,20,50$ and $100 \mu \mathrm{M})$. NAC incubation was started $1 \mathrm{~h}$ before addition of 3BP in order to saturate the cells with NAC. Control cells

that both have a myeloid leukemia origin. Here we further give a detailed analysis of various aspects of the THP-1 cells under different combined regimes of 3BP with DCA, lactate and $\mathrm{N}$-acetyl cysteine.

The flowcytometry data (Fig. 1) show a quite distinct reaction of the various cell lines on exposure to $3 \mathrm{BP}$, depending on the characteristics of the particular cell line. They all share the phenomenon of membrane depolarisation with increasing 3BP concentrations, whereas the main difference between these cells lines appears to be the induction of ROS production with increasing $3 \mathrm{BP}$ concentration. This latter property seems to be linked to the leukemia derived lines THP-1 and K562, with virtually no activity in the solid tumor derived lines MCF-7, ZR-75-1 and MDA-MB-435S.

Monocytes such as THP-1 cells are able to differentiate into macrophages, which have a functional ROS system in place to kill pathogens and clean up cellular debris by phagocytic activity (Mosser and Edwards 2008), and THP1 can be induced into macrophages by treatment with PMA (Tsuchija et al. 1982). K562 will differentiate into the erythroid direction when treated with hemin, upon which (TNF)-related apoptosis-inducing ligand (TRAIL) binding suffices to direct the cells to apoptosis (Hietakangas et al. 2003). Tumor cells, and especially those of solid tumors, do not have an active ROS system in place due to the hyperpolarization caused by HK2, which can be up-regulated 500 fold in liver tumor cells (Chen et al. 2009; Arora and are represented by the dot marked $0 \mathrm{BP}$. Two solid arrows indicate $0 \mathrm{BP}$ and 50BP ranges. The dotted lines connect dots of cells with the same NAC concentration, indicated on the 50BP arrow. Eigenvectors are indicated and marked where appropriate

Pedersen 1988). They merely rely on the aerobic glycolysis for their energy production (Pedersen 2007; Mosser and Edwards 2008). The key component of this process is assumed to be HK2, which is expressed in all malignant tumors, and to some degree, in healthy kidney cells (Kamel and Schwarzfischer 1975).

This is consistent with the observation that in any PET scan, the kidneys always show up as positive, regardless of their being a tumor in the kidneys (Chang et al. 2006). It would be a challenge to synthesize ${ }^{76} \mathrm{Br}$ (Ribeiro et al. 1999) labelled 3-bromopyruvate and perform a PET scan with it, to find whether it will co-locate with the signal of ${ }^{18} \mathrm{~F}$ deoxyglucose, as used in conventional PET scanning.

The levels of 3BP needed to cause membrane depolarisation and/or ROS induction differ for the lines tested in these experiments. K562 is the most resistant, showing no effects at all concentrations tested until complete collapse at $500 \mu \mathrm{M}$, whereas ZR-75-1, MDA-MB-435S and THP-1 already show signs of $3 \mathrm{BP}$ effects at $50 \mu \mathrm{M}$. MCF-7 was found to react at $100 \mu \mathrm{M}$, which is in good agreement with results in another laboratory (Fatima Balthazar, personal communication).

If the membrane depolarisation is the key action of 3BP, then it should be inducible even under circumstances where ROS activation is inhibited. This appears to be the case, as is clear from the data on the flowcytometry details (Fig. 2) and the combined NAC-3BP treatments (Fig. 5). It is well 
known that NAC is able to counter the effects of 3BP (Kim et al. 2008). This is also obvious from our data, but what remains is the reduction of the membrane potential, regardless of the concentration of NAC used, provided it is above the threshold level, as is shown by Fig. 5 .

The combination of $3 \mathrm{BP}$ with DCA and lactate has revealed that no concentration of DCA or lactate can abolish the ROS induction and membrane depolarisation by 3BP. This looks trivial, but recent research has suggested that membrane monocarboxylate transporters (MCT's) (Halestrap and Price 1999) are responsible for the accumulation of 3BP in tumor cells (Thangaraju et al. 2009), contrasting the mechanism by covalent binding to a cysteine in the active site of HK2, as shown by Pedersen (Pedersen 2007). The Km of this transporter isolated from rat liver cells and Ehrlich Lettre tumor cells from mouse shows an affinity for lactate and DCA of resp. 4.5 and 0.6 mM (Jackson and Halestrap 1996).

It seems remarkable that $3 \mathrm{BP}$ has such a profound effect on THP1 cells in the presence of 100-1,000 fold excess of DCA. Assuming that the excess of DCA completely saturates the monocarboxylate transporter MCT-1, 3BP cannot be transported into the cell and hence would show no effect. Figure 3 shows the effects of 3BP and DCA alone and in combination. This may lead to the conclusion that the cells do not carry MCT-1 or that 3BP circumvents MCT-1 binding and can enter independently of MCT-1.

Figure 4 also shows that the effect of $3 \mathrm{BP}$ on ROS production is almost constant, except for the DCA $5 \mathrm{mM}$ and both lactate co-treatments. In these cases, ROS stimulation was halved compared to all other treatments. The trends for zero BP (up to $5 \mathrm{mM} \mathrm{DCA}$ ) and 50 BP combined treatments were almost identical showing that the effects of the combination are additive, not synergistic. This implies that $3 \mathrm{BP}$ and DCA are working along different mechanisms, and that they could be combined safely, without any interfering complexities.

The NAC data show that some aspects of 3BP can be countered by the glutathione based defense system, as has been demonstrated with melanoma cell lines (Qin and Nickoloff 2010; Mannervik et al. 1987) and which is demonstrated in these experiments by the K562 cell line. This line has been demonstrated to be a glutathione S-transferase overexpressor (Yang et al. 2001), which makes it resistant to oxidative stress. K562 was the only cell line which did not give in to increasing $3 \mathrm{BP}$ concentration with regard to membrane depolarisation and ROS activity until $500 \mu \mathrm{M}$ was reached (Figs. 1 and 2). This is consistent with the observations in an experiment using melanoma cell lines which exhibited extreme resistance to $3 \mathrm{BP}$ treatment, until they are treated with L-buthionine, S,R,-sulfoximine. After pre-treatment with this compound at $1 \mathrm{mM}$ during $24 \mathrm{~h}$, all resistant cells were depleted in their glutathione levels and efficiently killed by subsequent $200 \mu \mathrm{M}$ 3BP treatment (Qin and Nickoloff 2010). This shows the power of the combination of inhibitors with different targets in the treatment of resistant tumors.

Our results show that the combination of THP-1 and K562 may serve as a useful model system to study the combination of 3BP and DCA on one hand, and possible resistance mechanisms due to glutathione on the other hand. The effects of DCA and 3BP on THP- 1 show that they are additive and not interfering with each other provided the concentrations used do not exert cytotoxic effects to healthy cells.

Open Access This article is distributed under the terms of the Creative Commons Attribution License which permits any use, distribution, and reproduction in any medium, provided the original author(s) and the source are credited.

\section{References}

Arora KK, Pedersen L (1988) Functional significance of mitochondrial bound hexokinase in tumorcell metabolism. J Biol Chem 263:17422-17428

Chang JM, Lee HJ, Jin Mo Goo JM, Lee HY, Lee JJ, Chung JK, Im JG (2006) False positive and false negative FDG-PET scans in various thoracic diseases. Korean J Radiol 7(1):57-69

Chen Z, Zhang H, Lu W, Huang P (2009) Role of mitochondriaassociated hexokinase II in cancer cell death induced by 3-bromopyruvate. Biochim Biophys Acta 1787:553-560

Dell'Antone P (2009) Targets of 3-bromopyruvate, a new, energy depleting, anticancer agent. Med Chem 5:491-496

Ellison G, Klinowska T, Westwood RF, Docter E, French T, Fox JC (2002) Further evidence to support the melanocytic origin of MDA-MB-435. Mol Pathol 55(5):294-299

Engel LW, Young NA, Tralka TS, Lippman ME, O’Brien SJ, Joyce MJ (1978) Establishment and characterization of three new continuous cell lines derived from human breast carcinomas. Cancer Res 38:3352-3364

Geschwind JF, Ko YH, Torbenson MS, Magee C, Pedersen PL (2002) Novel therapy for liver cancer: direct intraarterial injection of a potent inhibitor of ATP production. Cancer Res 62(14):39093913

Halestrap AP, Price NT (1999) The proton-linked monocarboxylate transporter (MCT) family: structure, function and regulation. Biochem J 343:281-299

Hietakangas V, Poukkula M, Heiskanen KM, Karvinen JT, Sistonen L, Eriksson JE (2003) Erythroid differentiation sensitizes K562 leukemia cells to TRAIL-induced apoptosis by downregulation of cFLIP. Mol Cell Biol 23(4):1278-1291

Jackson VN, Halestrap AP (1996) The kinetics, substrate, and inhibitor specificity of the monocarboxylate (lactate) transporter of rat liver cells determined using the fluorescent intracellular $\mathrm{pH}$ indicator 2', 7'bis(carboxymethyl)-5(6)-carboxyfluorescein. J Biol Chem 271:861-868

Kamel R, Schwarzfischer F (1975) Hexokinase isozymes in human neoplastic and fetal tissues: the existence of hexokinase II in malignant tumors and in placenta. Humangenetik 30:181-185

Kim JS, Ahn KJ, Kim JA, Kim HM, Lee JD, Lee JM, Kim SJ, Park JH (2008) Role of reactive oxygen species-mediated mitochondrial dysregulation in 3-bromopyruvate induced cell death in hepatoma cells: ROS-mediated cell death by 3-BrPA. J Bioenerg Biomembr 40(6):607-618 
Ko YH, Pedersen PL, Geschwind JF (2001) Glucose catabolism in the rabbit VX2 tumor model for liver cancer: characterization and targeting hexokinase. Cancer Lett 173(1):83-91

Ko YH, Smith BL, Wang Y, Pomper MG, Rini DA, Torbenson MS, Hullihen J, Pedersen PL (2004) Advanced cancers: eradication in all cases using 3-bromopyruvate therapy to deplete ATP. Biochem Biophys Res Commun 324(1):269-750

Lozzio CB, Lozzio BB (1975) Human chronic myelogenous leukemia cellline with positive Philadelphia chromosome. Blood 45(3):321-334

Mannervik B, Castro VM, Danielson UH, Tahir MK, Hansson J, Ringborg U (1987) Expression of class Pi glutathione transferase in human malignant melanoma cells. Carcinogenesis 8(12):19291932

Michelakis ED, Sutendra G, Dromparis P, Webster L, Haromy A, Niven E, Maguire C, Gammer TL, Mackey JR, Fulton D, Abdulkarim B, McMurtry MS, Petruk KC (2010) Metabolic modulation of glioblastoma with dichloroacetate. Sci Transl Med 2(31):31 ra$34 \mathrm{ra}$

Mosser DM, Edwards JP (2008) Exploring the full spectrum of macrophage activation. Nat Rev Immunol 8:958-969

Papandreou I, Goliasova T, Denko NC (2011) Anticancer drugs that target metabolism: is dichloroacetate the new paradigm? Int J Cancer 128:1001-1008

Pedersen PL (2007) Warburg, me and Hexokinase 2: multiple discoveries of key molecular events underlying one of cancers' most common phenotypes, the "Warburg Effect", i.e., elevated glycolysis in the presence of oxygen. J Bioenerg Biomembr 39(3):211-222

Porporato PE, Dhup S, Dadhich RK, Copetti T, Sonveaux P (2011) Anticancer targets in the glycolytic metabolism of tumors: a comprehensive review. Front Pharmacol 2:49. doi:10.3389/ fphar.2011.00049

Qin J-Z, Nickoloff BJ (2010) 3-Bromopyruvate induces necrotic cell death in sensitive melanoma cell lines. Biochem Biophys Res Commun 396:495-500
Ribeiro MJ, Almeida P, Strul D, Ferreira N, Loc'h C, Brulon V, Trébossen R, Mazière B, Bendriem B (1999) Comparison of fluorine-18 and bromine-76 imaging in positron emission tomography. Eur J Nucl Med 26(7):758-766

Soule HD, Vazquez J, Long A, Albert S, Brennan M (1973) A human cell line from a pleural effusion derived from a breast carcinoma. J Natl Cancer Inst 51(5):1409-1416

Stockwin LH, Yu SX, Borgel S, Hancock C, Wolfe TL, Phillips LR, Hollingshead MG, Newton DL (2010) Sodium dichloroacetate selectively targets cells with defects in the mitochondrial ETC. Int J Cancer 127(11):2510-2519

Sun RC, Fadia M, Dahlstrom JE, Parish CR, Board PG, Blackburn AC (2010) Reversal of the glycolytic phenotype by dichloroacetate inhibits metastatic breast cancer cell growth in vitro and in vivo. Breast Cancer Res Treat 120:253-260

Thangaraju M, Karunakaran SK, Itagaki S, Gopal E, Elangovan S, Prasad PD, Ganapathy V (2009) Transport via SLC5A8 with subsequent inhibition of histone deacetylases HDAC1 and HDAC3 underlies the antitumor activity of 3-Bromopyruvate. Cancer 115(20):4655-4666

Tsuchija S, Kobayashi Y, Goto Y, Okumura H, Nakae S, Konno T, Tada K (1982) Induction of maturation in cultured monocytic leukemia cells by a phorbol ester. Cancer Res 42:1530-1536

Tsuchiya S, Yamabe M, Yamaguchi Y, Kobayashi Y, Konno T, Tada K (1980) Establishment and characterization of a human acute monocytic leukemia cell line (THP-1). Int J Cancer 26:171-176

Warburg O, Wind F, Negelein E (1926) Ueber den Stoffwechsel von Tumoren im Körper. Klin Wochenschr 5:829-832

Yang Y, Cheng JZ, Singhal SS, Saini M, Pandya U, Awasthi S, Awasthi YC (2001) Role of glutathione S-transferases in protection against lipid peroxidation. Overexpression of hGSTH2-2 in K562 cells protects against hydrogen peroxide-induced apoptosis and inhibits JNK and caspase 3 activation. J Biol Chem 276:19220-19230 